\title{
MODELO MULTI-ESTÁGIO DE OTIMIZAÇÃO PARA O PLANEJAMENTO DA EXPANSÃO DE SISTEMAS DE DISTRIBUIÇÃO
}

\author{
Sérgio Haffner* \\ haffnerdieee.org \\ Luís Alberto Pereira* \\ lpereiradee.pucrs.br
}

\author{
Luís Fernando Pereira* \\ pereiradee.pucrs.br \\ Lucio Barreto* \\ barretodieee.org
}

\author{
${ }^{*}$ DEE-FENG-PUCRS \\ Av. Ipiranga, 6681 - Prédio 30 - Sala 150 \\ CEP 90619-900 - Porto Alegre RS
}

\begin{abstract}
This paper presents a multi-stage model for the problem of power distribution expansion planning. The expansion model considers the increase of substation capacities, installation of new substations and several alternatives for the feeders (enlargement, removing, merging, splitting, load transfer and conductor replacement). The objective function takes into account the present value of the total installation costs (feeders and substations) and the network operation and maintenance costs. The model also considers the operational constraints, concerning the equipment capacities and voltage limits, and logical constraints used to reduce the search space. In addition, the paper presents: (a) an extension of the linear disjunctive formulation, making possible the inclusion, exclusion and the replacement of the initial configuration branches; (b) a generalization of new paths constraints useful for complex network topologies. With the proposed mixed linear integer model the optimal solution can be achieved by means of mathematical programming methods, like the branch-and-bound algorithm. The validation and the efficiency of the proposed model are based on a medium voltage distribution network taken as an example of application.
\end{abstract}

\footnotetext{
Artigo submetido em 18/07/2006

1a. Revisão em 13/11/2006

2a. Revisão em 23/11/2006

Aceito sob recomendação do Editor Associado

Prof. Denis Vinicius Coury
}

KEYWORDS: power distribution, power distribution planning, power distribution economics.

\section{RESUMO}

Neste trabalho apresenta-se um modelo para o problema de planejamento em múltiplos estágios da expansão do sistema de distribuição de energia. O modelo de expansão considera a ampliação da capacidade das subestações existentes, a instalação de novas subestações e os diferentes tipos de alterações possíveis nos alimentadores (ampliação, remoção, união, subdivisão, transferência de carga e substituição de condutores). A função objetivo a ser minimizada representa o valor presente dos custos totais de instalação (alimentadores e subestações), de operação e manutenção da rede. O modelo apresentado considera restrições operacionais, relacionadas com a capacidade dos equipamentos e com os limites de tensão, e restrições lógicas, com o objetivo de reduzir o espaço de busca. São apresentadas: (a) uma extensão da formulação linear disjuntiva que é capaz de representar a inclusão, a exclusão e a substituição de ramos da configuração inicial; (b) uma generalização das restrições relacionadas com a criação de novos caminhos que pode ser aplicada em topologias mais complexas. O modelo linear inteiro misto resultante permite que a solução ótima seja obtida através de métodos de programação matemática, tais como o algoritmo branchand-bound. A validade e a eficiência do modelo apresentado são comprovadas por intermédio de um exemplo de aplica- 
ção em uma rede de distribuição em média tensão.

PALAVRAS-CHAVE: distribuição de energia, planejamento da expansão, otimização.

\section{INTRODUÇÃO}

O problema de planejamento da expansão do sistema de distribuição consiste em determinar a capacidade, a localização e o instante de instalação dos novos equipamentos de distribuição considerando as restrições de capacidade das linhas, queda de tensão e segurança no atendimento da demanda (Willis, 2004; Khator e Leung, 1997; Lakervi e Holmes, 1995; Temraz e Quintana, 1993).

Inicialmente, diversos autores tentaram resolver este problema de forma simplificada, considerando um modelo de planejamento estático para um determinado horizonte (Sun et alii, 1982; Aoki et alii, 1990). Destes trabalhos resultou a formalização do problema em um único estágio no qual os recursos necessários para o horizonte de planejamento são introduzidos de uma só vez. Geralmente utiliza-se um horizonte de curto prazo para que sejam selecionados os investimentos que correspondam às reais necessidades da rede, pois as incertezas nas previsões tendem a aumentar quando o horizonte se amplia.

Posteriormente, o problema foi adequado para considerar um horizonte de longo prazo (Gönen e Foote, 1982; RamírezRosado e Gönen, 1991; Nara et alii, 1992). Esta abordagem deu origem à formulação do problema em múltiplos estágios nos quais os recursos necessários para o horizonte de planejamento podem ser distribuídos de acordo com as necessidades previstas para cada estágio. Desta forma, as concessionárias podem acompanhar o crescimento gradual da demanda e realizar o planejamento a mínimo custo, considerando um horizonte de longo prazo. Os investimentos definidos para as etapas iniciais são efetivamente executados enquanto os investimentos definidos para as etapas finais são reavaliados no futuro considerando previsões atualizadas. Assim, o horizonte de planejamento desloca-se dinamicamente, de modo que o estágio inicial sempre coincida com o período (mês ou ano) de execução.

Os métodos de solução usados para resolver o problema de expansão podem ser divididos em duas categorias: métodos de programação matemática e métodos heurísticos, incluindo sistemas especialistas e algoritmos evolucionários. Entre os métodos de programação matemática destaca-se a programação inteira mista (Sun et alii, 1982; Vaziri et alii, 2004a e 2004b; Paiva et alii, 2005), a programação não-linear (Ponnavaikko et alii, 1987; El-Khattam et alii, 2005), a programação dinâmica (Boulaxis et alii, 2002; Díaz-Dorado e Pidre, 2004) e a programação linear (Farrag et alii, 1999). Com esta abordagem é possível representar explicitamente as principais restrições (Leis de Kirchhoff, capacidade dos equipamentos, queda de tensão e orçamento) e minimizar os custos fixos e variáveis relacionados com a instalação e substituição de equipamentos. Nas abordagens via programação inteira mista, a introdução de considerações práticas freqüentemente limita o número de soluções e torna os problemas combinatoriais associados computacionalmente tratáveis. Este fato, associado a possibilidade de garantia de otimalidade e a capacidade de processamento dos computadores disponíveis atualmente, torna esta abordagem muito atrativa.

Desde 1980, grandes esforços têm sido investidos na solução do problema de planejamento da distribuição empregando algoritmos heurísticos, que se tornaram uma alternativa aos métodos de programação matemática. Os métodos heurísticos ganharam espaço pela facilidade em considerar restrições e funções objetivos não-lineares, embora não existam garantias de que a solução ótima do problema seja obtida. Ainda, com esta abordagem é possível introduzir mais facilmente aspectos como perdas, confiabilidade e incertezas. Entre os métodos heurísticos destacam-se os algoritmos denominados "branch exchange" (Aoki et alii, 1990; Nara et alii, 1998; Míguez et alii, 2002) e os algoritmos baseados em computação evolucionária (Miranda et alii, 1994; RamírezRosado e Bernal-Augustín, 2001; Díaz-Dorado et alii, 2002). Outros métodos heurísticos também têm sido empregados na solução deste problema tais como os sistemas especialistas (Ranjan et alii, 2002), colônia de formigas (Gómez et alii, 2004), "simulated annealing" (Parada et alii, 2004) e busca tabu (Augugliaro et alii, 2002).

Propõe-se neste trabalho a solução ótima do problema de planejamento multi-estágio que emprega método de programação matemática e inclui características e restrições usualmente empregadas nas abordagens heurísticas. O modelo considera os diversos tipos de alterações possíveis nos nós e nos ramos das redes de distribuição e as diversas restrições operacionais e financeiras do problema de expansão. As alterações associadas aos nós incluem: instalação de novas subestações, ampliação de subestações existentes, instalação de novos transformadores de força e corte de carga. As alterações associadas aos ramos incluem: instalação, alteração e remoção de trechos de alimentadores com opção por diferentes bitolas. A possibilidade de representação destas alternativas de alteração e a consideração de um horizonte de longo prazo (dividido em estágios) permitem que se obtenham custos finais inferiores aos obtidos pelos modelos mais simples, onde apenas parte destas alternativas pode ser simultaneamente analisada. Apresenta-se, também, uma extensão da abordagem disjuntiva tipicamente empregada na linearização dos problemas relacionados com o planejamento da expansão dos sistemas de transmissão empregando o modelo de rede do fluxo de carga DC (Bahiense et alii, 2001; Ro- 
mero et alii, 2002). Na formulação dos problemas de expansão da transmissão e distribuição as não-linearidades nas restrições estão relacionadas com o produto entre as variáveis binárias e variáveis contínuas. Na abordagem clássica apenas a inclusão ou não de um ramo é representada pela formulação disjuntiva; na abordagem apresentada neste trabalho representa-se também a remoção e a substituição dos ramos.

Para reduzir o espaço de busca do problema inteiro misto que descreve o planejamento da expansão, são introduzidas: (a) restrições lógicas, que descrevem limitações de investimento; (b) restrições de cerca, obtidas a partir da Lei de Kirchhoff das correntes; (c) restrições de novos caminhos (Monticelli et alii, 1982), que são generalizadas para poder operar com topologias mais complexas.

O artigo está organizado de acordo com a seguinte seqüência. Na seção 2, é apresentada a notação. Na seção 3, apresentase a modelagem do problema de otimização, sendo apresentadas em detalhes a função objetivo e as restrições empregadas. Na seção 4, descreve-se o modelo de carga e da rede. Na seção 5 apresenta-se um exemplo de aplicação do modelo proposto considerando o planejamento em três estágios. Para finalizar são apresentadas as conclusões.

\section{NOTAÇÃO}

\section{Conjuntos}

$\Omega^{F}$ e $\Omega_{i}^{F}$ ramos da rede fixa (ramos pré-definidos ao longo do horizonte de planejamento) e alterações definidas para cada ramo $i$ da rede fixa

$\Omega^{R}$ e $\Omega_{j}^{R}$ ramos da rede com alternativas de substituição e alternativas de substituição candidatas do ramo $j$

$\Omega^{A}$ e $\Omega_{k}^{A}$ ramos da rede com alternativas de adição e alternativas de adição candidatas do ramo $k$

$\Omega^{S}$ e $\Omega_{l}^{S}$ nós da rede relacionados com as subestações existentes e alternativas de instalação ou ampliação e alternativas de adição ou ampliação do nó $l$

$\Omega^{D}$ nós da rede que possuem demanda

Variáveis binárias de investimento $(x=1$ significa que a alternativa será selecionada)

$x_{j, t}^{R J}$ variável associada à substituição (recondutoração) pela alternativa $R J$ do ramo $j$ no estágio $t$

$x_{k, t}^{A K}$ variável associada à adição da alternativa $A K$ do ramo $k$ no estágio $t$ $x_{l, t}^{S 0}$ variável associada aos custos fixos de instalação ou ampliação de uma subestação no nó $l$ no estágio $t$

$x_{l, t}^{S L}$ variável associada a ampliação pela alternativa $S L$ da subestação do nó $l$ no estágio $t$

$\mathbf{x}_{t}$ vetor das variáveis de investimento do estágio $t$

Variáveis binárias de utilização e seus limites ( $y=1$ significa que a alternativa será utilizada)

$y_{i, t}^{F I}$ e $y_{\max , i, t}^{F I}$ variável associada à utilização da alteração definida $F I$ do ramo $i$ da rede fixa no estágio $t$ e seu limite máximo: $y_{\max , i, t}^{F I}=1$ significa que a alteração FI do ramo $i$ está disponível para utilização no estágio $t$

$y_{j, t}^{R 0} \mathbf{e} y_{j, t}^{R J}$ variável associada à utilização do ramo inicial $R 0$ ou da alternativa $R J$ de substituição do ramo $j$ no estágio $t$

$y_{k, t}^{A K}$ variável associada à utilização da alternativa de adição $A K$ do ramo $k$ no estágio $t$

\section{Variáveis contínuas e seus limites}

$f_{i, t}^{F} \mathbf{e} f_{\max , i}^{F I}$ fluxo de corrente no ramo $i$ da rede fixa no estágio $t$ e capacidade máxima da alteração $F I$ definida para o respectivo ramo

$f_{j, t}^{R}, f_{\text {max }, j}^{R 0}$ e $f_{\text {max }, j}^{R J}$ fluxo de corrente no ramo $j$ da rede de substituição no estágio $t$ e capacidades máximas inicial $R 0$ e da alternativa $R J$ do respectivo ramo

$f_{k, t}^{A}$ e $f_{\max , k}^{A K}$ fluxo de corrente no ramo $k$ da rede de adição no estágio $t$ e capacidade máxima da alternativa $A K$ do respectivo ramo

$\mathbf{f}_{t}^{F}, \mathbf{f}_{t}^{R} \mathbf{e} \mathbf{f}_{t}^{A}$ vetores coluna dos fluxos de corrente nos ramos da rede fixa e das redes candidatas à substituição e à adição no estágio $t$

$\mathbf{g}_{l, t}^{S}, \mathbf{g}_{\max , l, t}^{S} \mathbf{e} \mathbf{g}_{\max , l}^{S L}$ injeção de corrente no nó $l$ no estágio $t$ e seus limites máximos da capacidade existente (no estágio $t$ ) e da alternativa $S L$ de instalação ou ampliação de subestações

$r_{m, t}$ injeção fictícia de corrente (corte de carga) no nó $m$ no estágio $t$

$\mathbf{r}_{t}$ vetor coluna das injeções fictícias de corrente nodais (corte de carga) no estágio $t$

$\mathbf{g}_{t}$ vetor coluna das injeções de corrente nodais no estágio $t$ 
$\mathbf{V}_{t}, \mathbf{V}_{\min } \mathbf{e} \mathbf{V}_{\max }$ vetor coluna das magnitudes das tensões nodais no estágio $t$ e seus limites mínimo e máximo

$c_{t}^{i n v}\left(\mathbf{x}_{t}\right)$ custo de investimento do estágio $t$

$c_{t}^{\text {oper }}\left(\mathbf{r}_{t}, \mathbf{g}_{t}\right)$ custo de operação do estágio $t$

\section{Parâmetros}

$d_{m, t}$ demanda de corrente nodal (carga) no nó $m$ no estágio $t$

$\mathbf{d}_{t}$ vetor coluna das demandas de corrente nodais (cargas) no estágio $t$

$\mathbf{S}^{F}$ matriz incidência nó-ramo da rede fixa

$\mathbf{S}^{R}$ matriz incidência nó-ramo da rede com alternativas de substituição

$\mathbf{S}^{A}$ matriz incidência nó-ramo da rede com alternativas de adição

$Z_{i}^{F I}$ impedância da alteração definida $F I$ do ramo $i$ da rede fixa

$Z_{j}^{R 0}$ e $Z_{j}^{R J}$ impedâncias inicial e da alternativa de substituição $R J$ do $\operatorname{ramo} j$

$Z_{k}^{A K}$ impedância da alternativa de adição $A K$ do ramo $k$

$M$ número grande o bastante para relaxar as restrições disjuntivas

$N_{t}$ número de nós da rede no estágio $t$, excluindo subestações

$\delta_{t}^{i n v} \mathbf{e} \delta_{t}^{\text {oper }}$ fatores utilizados para converter em valor presente os custos de investimento e de operação, respectivamente, no estágio $t$

$O_{i}^{F I}$ custo de operação da alteração definida $F I$ do ramo $i$

$C_{j}^{R J}$ e $O_{j}^{R J}$ custos de investimento e de operação da alternativa de substituição $R J$ do ramo $j$

$C_{k}^{A K}$ e $O_{k}^{A K}$ custos de investimento e de operação da alternativa de adição $A K$ do ramo $k$

$C_{l}^{S 0}$ custo fixo de instalação ou ampliação de uma subestação no nó $l$

$C_{l}^{S L}$ custo da alternativa $S L$ de ampliação da subestação do nó $l$

$C_{m}^{D}$ custo da energia não suprida do nó $m$

$B$ e $B_{t}$ orçamento total disponível e orçamento disponível no estágio $t$
$T$ número de estágios do horizonte de planejamento

$I$ taxa de juros por período de tempo

$p(t)$ número de períodos de tempo do estágio $t$ a partir de um referencial (mês ou ano) adotado como base

$\Delta p(t)$ duração em períodos de tempo do estágio $t$

\section{FORMULAÇÃO DO PROBLEMA}

O problema da expansão do sistema de distribuição considerando um horizonte de longo prazo foi modelado levando em conta os seguintes fatores:

- a rede de distribuição é dividida em nós, nos quais as cargas e fontes são concentradas, e ramos que realizam a conexão entre os nós, representando o percurso dos alimentadores;

- o horizonte de planejamento é dividido em $T$ estágios com duração conhecida, sendo as demais variáveis do problema associadas a cada um destes estágios;

- a cada nó são associadas duas variáveis: uma representa o módulo da tensão nodal e a outra a injeção de corrente; a cada ramo é associada uma variável que representa o respectivo fluxo de corrente;

- em qualquer um dos estágios do horizonte considerado, são permitidas alterações nos nós (ampliação da capacidade e instalação de novas subestações) e nos ramos (substituição do condutor de um ramo pré-existente e adição de um ramo em um trecho onde não havia ligação prévia);

- as possibilidades de alteração nos nós e nos ramos da rede de distribuição constituem o conjunto das alternativas de investimento que serão empregadas para solução do problema de expansão;

- a execução de cada uma das alternativas de investimento ao longo dos estágios está associada a uma variável binária $x$, que assume o valor unitário quando a alternativa for selecionada no estágio $t$ e o valor zero quando a alternativa não for selecionada;

- a cada tipo de alteração estão associados custos de investimento para substituição de ramo $\left(C_{j}^{R J}\right)$; adição de um novo ramo $\left(C_{k}^{A K}\right)$; ampliação e instalação de uma subestação no nó $\left(C_{l}^{S O}\right.$ e $\left.C_{l}^{S L}\right)$;

- a utilização dos ramos disponíveis na rede de distribuição está associada a variáveis binárias $y$, que assumem valor unitário quando as alternativas forem utilizadas no estágio $t$ e o valor zero quando as alternativas não forem selecionadas; 
- para todos os ramos da rede estão associados custos de operação e manutenção $\left(O_{i}^{F I}, O_{j}^{R 0}, O_{j}^{R J}\right.$ e $\left.O_{k}^{A K}\right)$;

- as injeções e tensões nodais e os fluxos nos ramos são determinados através das duas Leis de Kirchhoff, definidas para todos os estágios;

- a carga é representada por injeções constantes de corrente com valores determinados para cada estágio;

- são considerados os limites de capacidade dos condutores, as capacidades das subestações e a disponibilidade da geração distribuída em todos os estágios;

- as quedas de tensão na rede de distribuição são calculadas a partir do produto entre o fluxo de corrente e a impedância das linhas em cada estágio;

- a função objetivo corresponde a minimização do valor presente dos custos de investimento e de operação;

- são considerados limites de investimento total $(B) \mathrm{e}$ para cada estágio $t\left(B_{t}\right)$.

As variáveis associadas às alterações definidas para o ramo $i$ da rede fixa são caracterizadas pelo índice $F I$. As variáveis associadas com as possibilidades de alteração do ramo $j$ da rede de substituição são caracterizadas pelo índice $R J$. As variáveis associadas com as possibilidades de alteração do ramo $k$ da rede de adição são caracterizadas pelo índice $A K$. As variáveis associadas com as alternativas de expansão da subestação localizada no nó $l$ são caracterizadas pelo índice $S L$. Com esta representação, é possível definir o número de alterações associadas a cada ramo (ou nó) da rede, independentemente do número de alterações definidas para os outros ramos (ou nós). Assim, alguns ramos da rede podem ter apenas uma alternativa de alteração, outros duas alternativas, outros três alternativas e assim sucessivamente.

As variáveis que representam se os investimentos devem ser selecionados são agrupadas de acordo com o tipo de alteração associada: substituição do condutor de um ramo existente (variáveis $x_{j, t}^{R J}$ ); adição de um novo trecho de alimentador em um ramo (variáveis $x_{k, t}^{A K}$ ); instalação de uma nova subestação ou ampliação da capacidade de uma subestação existente em um nó (variáveis $x_{l, t}^{S}$ e $x_{l, t}^{S L}$, respectivamente). Para cada um dos estágios, a utilização ou não dos recursos disponíveis nos ramos da rede (pré-existentes ou previamente instalados) está associada ao valor de variáveis binárias $\left(y_{i, t}^{F I}, y_{j, t}^{R J}\right.$ e $\left.y_{k, t}^{A K}\right)$ que assumem o valor unitário quando a respectiva alternativa for utilizada no estágio $t$.

As alterações definidas a priori nos ramos da rede (adição, substituição ou remoção) são facilmente incorporadas ao modelo por intermédio dos limites das variáveis de utilização

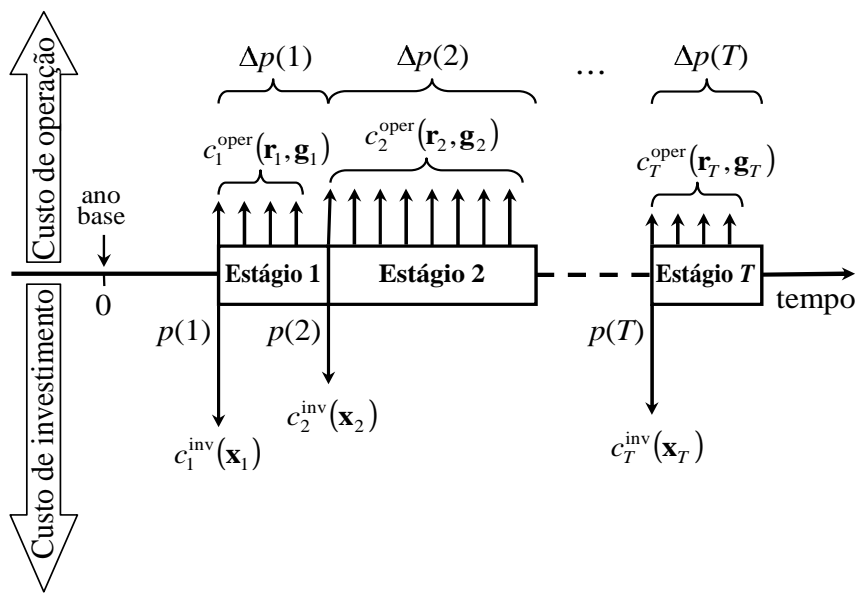

Figura 1: Instantes de efetivação dos custos de operação e de investimento no problema multi-estágio

dos ramos da rede fixa $\left(y_{\text {max }, i, t}^{F I}\right)$ definidos para cada estágio. Por exemplo, caso a alternativa $F 2$ do ramo 5 seja efetivada no terceiro estágio, os limites serão dados por: $y_{\max , 5,1}^{F 2}=y_{\max , 5,2}^{F 2}=0$ e $y_{\max , 5,3}^{F 2}=1$. Isto impossibilita o uso da alternativa $F 2$ nos dois primeiros estágios. De forma similar, as alterações definidas a priori nos nós da rede (capacidade das subestações) podem ser introduzidas no modelo por intermédio dos limites da capacidade existente $\left(\mathbf{g}_{\max , l, t}^{S}\right)$ que são definidos para cada estágio.

Assim, o problema geral do planejamento da expansão da rede de distribuição corresponde a um problema de programação inteira mista (PIM), conforme definido a seguir.

\subsection{Função objetivo}

A função objetivo do problema possui duas partes: custo de investimento $\left(c_{t}^{\text {inv }}\left(\mathbf{x}_{t}\right)\right)$ e custo de operação $\left(c_{t}^{\text {oper }}\left(\mathbf{r}_{t}, \mathbf{g}_{t}\right)\right)$, conforme apresentado na figura 1. O custo de investimento é efetivado no início de cada estágio e corresponde aos custos associados às alterações nos ramos (mudança de bitola de seções de alimentadores pré-existentes ou instalação de novas seções de alimentadores, denotados por $C_{j}^{R J}$ e $C_{k}^{A K}$ ) e nos nós da rede (ampliação de subestações pré-existentes ou instalação de novas subestações, denotados por $C_{l}^{S 0}$ e $\left.C_{l}^{S L}\right)$. O custo de operação é considerado no início de cada período de tempo do estágio e corresponde ao custo anual de operação e manutenção dos ramos em uso da rede de distribuição $\left(O_{i}^{F I}, O_{j}^{R 0}, O_{j}^{R J}\right.$ e $\left.O_{k}^{A K}\right)$ e da energia não suprida $\left(C_{m}^{D}\right)$.

Para um horizonte de planejamento dividido em $T$ estágios, a função objetivo corresponde à minimização do valor presente dos custos que são distribuídos ao longo do tempo, sendo 
dada pela seguinte expressão:

$$
\begin{aligned}
& C(\mathbf{x}, \mathbf{r}, \mathbf{g})=\sum_{t=1}^{T}\left[\delta_{t}^{\text {inv }} c_{t}^{\text {inv }}\left(\mathbf{x}_{t}\right)+\delta_{t}^{\text {oper }} c_{t}^{\text {oper }}\left(\mathbf{r}_{t}, \mathbf{g}_{t}\right)\right] \\
& c_{t}^{\mathrm{inv}}\left(\mathbf{x}_{t}\right)=\sum_{j \in \Omega^{R}} \sum_{J \in \Omega_{j}^{R}} C_{j}^{R J} x_{j, t}^{R J}+ \\
& +\sum_{k \in \Omega^{A}} \sum_{K \in \Omega_{k}^{A}} C_{k}^{A K} x_{k, t}^{A K}+ \\
& +\sum_{l \in \Omega^{S}}\left(C_{l}^{S 0} x_{l, t}^{S 0}+\sum_{L \in \Omega_{l}^{S}} C_{l}^{S L} x_{l, t}^{S L}\right) \\
& c_{t}^{\text {oper }}\left(\mathbf{r}_{t}, \mathbf{g}_{t}\right)=\sum_{i \in \Omega^{F}} \sum_{I \in \Omega_{i}^{F}} O_{i}^{F I} y_{i, t}^{F I}+ \\
& +\sum_{j \in \Omega^{R}}\left[O_{j}^{R 0} y_{j, t}^{R 0}+\sum_{J \in \Omega_{j}^{R}} O_{j}^{R J} y_{j, t}^{R J}\right]+ \\
& +\sum_{k \in \Omega^{A}} \sum_{K \in \Omega_{k}^{A}} O_{k}^{A K} y_{k, t}^{A K}+\sum_{m \in \Omega^{D}} C_{m}^{D} r_{m, t} \\
& \delta_{t}^{\text {inv }}=\frac{1}{(1+I)^{p(t)}} \\
& \delta_{t}^{\text {oper }}=\sum_{p=p(t)}^{p(t)+\Delta p(t)-1} \frac{1}{(1+I)^{p}}
\end{aligned}
$$

A utilização das injeções fictícias $r_{m, t}$ para representar os cortes de carga permite que sempre existam soluções factíveis, mesmo quanto são impostas restrições de investimento. Desta forma, estas restrições podem ser definidas para cada um dos estágios e para todo o horizonte de planejamento sem comprometer a factibilidade do problema.

\subsection{Restrições}

As restrições do problema estão divididas em quatro blocos e são oriundas da aplicação das Leis de Kirchhoff e da imposição dos limites operacionais dos equipamentos e dos recursos disponíveis (limitações financeiras) em todos os estágios do horizonte de planejamento. O primeiro bloco de restrições é obtido com a imposição da Lei de Kirchhoff das Correntes (LKC) para todos os estágios do horizonte:

$$
\mathbf{S}^{F} \mathbf{f}_{t}^{F}+\mathbf{S}^{R} \mathbf{f}_{t}^{R}+\mathbf{S}^{A} \mathbf{f}_{t}^{A}+\mathbf{g}_{t}+\mathbf{r}_{t}=\mathbf{d}_{t} \forall t=1, \cdots, T
$$

O segundo bloco de restrições advém da aplicação da Lei de
Kirchhoff das Tensões (LKT), para todo $t=1, \cdots, T$. Considerando a rede composta por ramos fixos, ramos candidatos à substituição e ramos que podem ser adicionados para composição final da rede, tem-se:

$$
\begin{aligned}
Z_{i}^{F I} f_{i, t}^{F}+\left[\mathbf{S}^{F}\right] \underset{\text { linha } i}{\mathrm{~T}} \mathbf{V}_{t}=0 \\
\qquad\left\{i \in \Omega^{F}, I \in \Omega_{i}^{F} \mid y_{i, t}^{F I}=1\right\}
\end{aligned}
$$

$$
\begin{array}{r}
Z_{j}^{R 0} f_{j, t}^{R}+\left[\mathbf{S}^{R}\right]_{\text {linha } j}^{\mathrm{T}} \mathbf{V}_{t}=0 \\
\left\{\forall j \in \Omega^{R} \mid y_{j, t}^{R 0}=1\right\} \\
Z_{j}^{R J} f_{j, t}^{R}+\left[\mathbf{S}^{R}\right] \stackrel{\text { linha } j}{\mathrm{~T}} \mathbf{V}_{t}=0 \\
\left\{\forall j \in \Omega^{R}, J \in \Omega_{j}^{R} \mid y_{j, t}^{R J}=1\right\} \\
Z_{k}^{A K} f_{k, t}^{A}+\left[\mathbf{S}^{A}{ }_{\operatorname{linha} k}^{\mathrm{T}} \cdot \mathbf{V}_{t}=0\right. \\
\left\{\forall k \in \Omega^{A}, K \in \Omega_{k}^{A} \mid y_{k, t}^{A K}=1\right\}
\end{array}
$$

onde o superescrito T indica matriz transposta. Deve-se observar que a existência das restrições (3) a (6) depende do valor assumido pelas variáveis de utilização $y_{i, t}^{F I}, y_{j, t}^{R 0}, y_{j, t}^{R J} \mathrm{e}$ $y_{k, t}^{A K}$, que determinam se os recursos serão utilizados ou não. Tais restrições só existem quando a respectiva variável de utilização assume valor unitário (indicando sua utilização nesta etapa), sendo a implementação desta dependência realizada pela multiplicação da restrição pela variável correspondente. Assim, surgem não-linearidades no modelo, pois as variáveis de utilização multiplicam os fluxos $\left(f_{i, t}^{F}, f_{j, t}^{R}\right.$ e $\left.f_{k, t}^{A}\right)$ e as tensões $V_{t}$. Para contornar esta não-linearidade adotouse uma extensão do modelo linear disjuntivo já empregado com sucesso no planejamento da expansão dos sistemas de transmissão (Bahiense et alii, 2001, Romero et alii, 2002), constituindo uma proposta inovadora na formulação do problema de planejamento da expansão para a área de distribuição. Quando formulado desta maneira o problema resultante é linear e pode ser resolvido diretamente por meio de métodos de otimização clássica sem necessidade de aplicar decomposições ou métodos heurísticos. Na aplicação proposta neste trabalho, são introduzidas ainda as seguintes funcionalidades:

- para um mesmo ramo são consideradas diversas possibilidades de configuração (diferentes bitolas e tipos de estruturas), sendo selecionada apenas a mais indicada;

- em qualquer estágio é possível considerar a remoção dos ramos que se tornam obsoletos (seccionamento de alimentadores). 
Assim, as equações (3) a (6) são substituídas pelas suas versões disjuntivas, dadas por:

$$
\begin{aligned}
& \left|Z_{i}^{F I} f_{i, t}^{F}+\left[\mathbf{S}^{F}\right]_{\text {linha } i}^{\mathrm{T}} \mathbf{V}_{t}\right| \leq M\left(1-y_{i, t}^{F I}\right) \\
& \left\{\forall i \in \Omega^{F}, I \in \Omega_{i}^{F}\right\} \\
& \left|Z_{j}^{R 0} f_{j, t}^{R}+\left[\mathbf{S}^{R}\right] \stackrel{\mathrm{T}}{\text { linha } j} \mathbf{V}_{t}\right| \leq M\left(1-y_{j, t}^{R 0}\right) \\
& \left\{\forall j \in \Omega^{R}\right\} \\
& \left|Z_{j}^{R J} f_{j, t}^{R}+\left[\mathbf{S}^{R}\right]_{\text {linha } j}^{\mathrm{T}} \mathbf{V}_{t}\right| \leq M\left(1-y_{j, t}^{R J}\right) \\
& \left\{\forall j \in \Omega^{R}, J \in \Omega_{j}^{R}\right\} \\
& \left|Z_{k}^{A K} f_{k, t}^{A}+\left[\mathbf{S}^{A}\right]_{\text {linha } k}^{\mathrm{T}} \mathbf{V}_{t}\right| \leq M\left(1-y_{k, t}^{A K}\right) \\
& \left\{\forall k \in \Omega^{A}, K \in \Omega_{k}^{A}\right\}
\end{aligned}
$$

Nas expressões (3.1) a (6.1), quando a variável $y$ assume valor nulo, a respectiva restrição é relaxada, pois $M$ é grande o bastante para que ambas desigualdades sempre sejam satisfeitas para os valores possíveis de $V_{t}$. Por outro lado, quando a variável $y$ assume valor unitário, as desigualdades (3.1) a (6.1) operam da mesma forma que as respectivas igualdades das expressões (3) a (6).

O terceiro bloco de restrições inclui os limites operacionais dos equipamentos e os limites de investimento. Os limites de fluxo nos ramos para $t=1, \cdots, T$ dependem da utilização dos recursos disponíveis e são dados por:

$$
\begin{gathered}
\left|f_{i, t}^{F}\right| \leq \sum_{I \in \Omega_{i}^{F}} y_{i, t}^{F I} f_{\max , i}^{F I} \quad\left\{\forall i \in \Omega^{F}\right\} \\
\left|f_{j, t}^{R}\right| \leq y_{j, t}^{R 0} f_{\max , j}^{R 0}+\sum_{J \in \Omega_{j}^{R}} y_{j, t}^{R J} f_{\max , j}^{R J} \quad\left\{\forall j \in \Omega^{R}\right\} \\
\left|f_{k, t}^{A}\right| \leq \sum_{K \in \Omega_{k}^{A}} y_{k, t}^{A K} f_{\max , k}^{A K} \quad\left\{\forall k \in \Omega^{A}\right\}
\end{gathered}
$$

Para as subestações próprias, os limites de injeção para $t=$ $1, \cdots, T$ dependem da capacidade disponível e dos investimentos em adição e ampliação realizados até cada estágio, sendo dados por:

$$
0 \leq \mathbf{g}_{l, t}^{S} \leq \mathbf{g}_{\max , l, t}^{S}+\sum_{L \in \Omega_{l}^{S}}\left(\sum_{\tau=1}^{t} x_{l, \tau}^{S L}\right) \mathbf{g}_{\max , l}^{S L}\left\{\quad \forall l \in \Omega^{S}\right\}
$$

Os demais limites operacionais são os valores máximos possíveis de corte de carga nas barras com demanda e a faixa de valores aceitáveis para a magnitude da tensão nodal nas subestações e nos nós onde existem cargas ou geração instaladas, para $t=1, \cdots, T$ :

$$
\begin{gathered}
0 \leq r_{m, t} \leq d_{m, t} \quad\left\{\forall m \in \Omega^{D}\right\} \\
V_{\text {min }, l} \leq V_{l, t} \leq V_{\text {max }, l} \quad\left\{\forall l \in \Omega^{S}\right\} \\
V_{\min , m} \leq V_{m, t} \leq V_{\max , m} \quad\left\{\forall m \in \Omega^{D}\right\}
\end{gathered}
$$

Os limites de investimento são considerados para cada estágio e para todo horizonte de planejamento, por intermédio das seguintes expressões:

$$
\begin{aligned}
& \sum_{j \in \Omega^{R}} \sum_{J \in \Omega_{j}^{R}} C_{j}^{R J} x_{j, t}^{R J}+\sum_{k \in \Omega^{A}} \sum_{K \in \Omega_{k}^{A}} C_{k}^{A K} x_{k, t}^{A K}+ \\
+ & \sum_{l \in \Omega^{S}}\left(C_{l}^{S 0} x_{l, t}^{S 0}+\sum_{L \in \Omega_{l}^{S}} C_{l}^{S L} x_{l, t}^{S L}\right) \leq B_{t} \quad \forall t=1, \cdots, T
\end{aligned}
$$

$$
\begin{aligned}
& \sum_{t=1}^{T}\left[\delta _ { t } ^ { \operatorname { i n v } } \left(\sum_{j \in \Omega^{R}} \sum_{J \in \Omega_{j}^{R}} C_{j}^{R J} x_{j, t}^{R J}+\right.\right. \\
& +\sum_{k \in \Omega^{A}} \sum_{K \in \Omega_{k}^{A}} C_{k}^{A K} x_{k, t}^{A K}+ \\
& \left.\left.+\sum_{l \in \Omega^{S}}\left(C_{l}^{S 0} x_{l, t}^{S 0}+\sum_{L \in \Omega_{l}^{S}} C_{l}^{S L} x_{l, t}^{S L}\right)\right)\right] \leq B
\end{aligned}
$$

O quarto bloco consiste nas restrições lógicas do problema, escritas em função das variáveis de investimento e utilização, sendo formado pelas seguintes restrições:

- para evitar trocas de condutores ao longo do horizonte, só é permitida uma alteração por ramo candidato à substituição ou adição;

$$
\begin{aligned}
& \sum_{t=1}^{T} \sum_{J \in \Omega_{j}^{R}} x_{j, t}^{R J} \leq 1 \quad\left\{\forall j \in \Omega^{R}\right\} \\
& \sum_{t=1}^{T} \sum_{K \in \Omega_{k}^{A}} x_{k, t}^{A K} \leq 1 \quad\left\{\forall k \in \Omega^{A}\right\}
\end{aligned}
$$

- ao longo do horizonte de planejamento, cada um dos investimentos em adição e ampliação de subestações pode ser realizado apenas uma vez; 


$$
\begin{gathered}
\sum_{t=1}^{T} x_{l, t}^{S} \leq 1 \quad\left\{\forall l \in \Omega^{S}\right\} \\
\sum_{t=1}^{T} x_{l, t}^{S L} \leq 1 \quad\left\{\forall l \in \Omega^{S}, L \in \Omega_{l}^{S}\right\}
\end{gathered}
$$

- os investimentos em adição e ampliação de subestações só podem ser executados após a realização da decisão pelos custos fixos que não implicam aumento de capacidade;

$$
\begin{aligned}
x_{l, t}^{S L} \leq \sum_{\tau=1}^{t} x_{l, \tau}^{S} \quad \forall t= & 1, \cdots, T \\
& \left\{\forall l \in \Omega^{S}, L \in \Omega_{l}^{S}\right\}
\end{aligned}
$$

- a rede fixa só poderá ser utilizada quando disponível;

$$
\begin{aligned}
0 \leq y_{i, t}^{F I} \leq y_{\max , i, t}^{F I} \quad \forall t & =1, \cdots, T \\
& \left\{\forall i \in \Omega^{F}, I \in \Omega_{i}^{F}\right\}
\end{aligned}
$$

- os ramos candidatos à substituição só podem ser utilizados após a realização do respectivo investimento;

$$
\begin{aligned}
y_{j, t}^{R J} \leq \sum_{\tau=1}^{t} x_{j, \tau}^{R J} \quad \forall t= & 1, \cdots, T \\
& \left\{\forall j \in \Omega^{R}, J \in \Omega_{j}^{R}\right\}
\end{aligned}
$$

- o investimento em qualquer alternativa de substituição exclui a possibilidade de utilização da configuração inicial prevista para aquele ramo;

$$
\begin{array}{r}
y_{j, t}^{R 0} \leq 1-\sum_{\tau=1}^{t} \sum_{J \in \Omega_{j}^{R}} x_{j, \tau}^{R J} \quad \forall t=1, \cdots, T \\
\left\{\forall j \in \Omega^{R}\right\}
\end{array}
$$

- os ramos candidatos à adição só podem ser utilizados após a realização do respectivo investimento;

$$
\begin{aligned}
y_{k, \tau}^{A K} \leq \sum_{t=1}^{\tau} x_{k, t}^{A K} \quad \forall t & =1, \cdots, T \\
& \left\{\forall k \in \Omega^{A}, K \in \Omega_{k}^{A}\right\}
\end{aligned}
$$

- evitar formação de malhas;

$$
\begin{aligned}
& \sum_{k \in \Omega^{A}} \sum_{K \in \Omega_{k}^{A}} y_{k, t}^{A K}+\sum_{i \in \Omega^{F}} \sum_{I \in \Omega_{i}^{F}} y_{i, t}^{F I}+ \\
&+\sum_{j \in \Omega^{R}}\left(y_{j, t}^{R 0}+\sum_{J \in \Omega_{j}^{R}} y_{j, t}^{R J}\right) \leq N_{t} \\
& \forall t=1, \cdots, T
\end{aligned}
$$

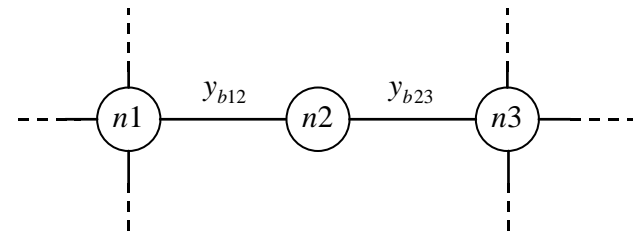

Figura 2: Novo caminho definido por um nó de passagem com dois circuitos adjacentes

\subsection{Restrições adicionais}

Além das restrições anteriores, é possível introduzir um conjunto de restrições adicionais baseadas no conhecimento das redes de energia elétrica. Neste trabalho foram utilizadas duas classes de restrições, relacionadas com a adição de novos caminhos (Monticelli et alii, 1982) e com a adição de restrições de cerca (Baldwin et alii, 1960).

\subsubsection{Restrições de novos caminhos}

Um novo caminho consiste de dois ou mais segmentos de rede conectados em série que constituem um circuito que conecta dois ou mais nós da rede de distribuição. Durante o processo de solução as variáveis associadas a um mesmo caminho são usadas de forma independente, sendo sua natureza complementar ignorada. Entretanto, a utilização dos segmentos que constituem um caminho só traz benefício para a rede quando uma combinação adequada de seus componentes é simultaneamente utilizada, pois a falta de um único componente interrompe o circuito série.

A forma de determinação e funcionamento das restrições de novos caminhos pode ser ilustrada através do exemplo simplificado da figura 2, na qual o nó de passagem $n 2$ (sem carga ou geração conectada) apresenta apenas dois ramos adjacentes, sendo as variáveis de utilização representadas pelas variáveis binárias $y_{b 12}$ e $y_{b 23}$, respectivamente, para os ramos $b 12$ e $b 23$.

Por inspeção do diagrama da figura 2, observa-se que a utilização em separado do ramo $b 12$ ou $b 23$ não tem utilidade, pois o nó $n 2$ não tem carga ou geração conectada. Quando estes ramos são utilizados simultaneamente, o percurso $n 1$ $n 2-n 3$ constitui um caminho. Assim, as alternativas de utilização deste caminho devem respeitar a seguinte restrição, associada ao nó de passagem $n 2$ :

$$
y_{b 12}=y_{b 23}
$$

Quando o nó de passagem $n 2$ apresenta três ramos adjacentes conforme mostrado na figura 3 , existem $2^{3}$ combinações de utilização dos ramos adjacentes, sendo que três delas não tem 


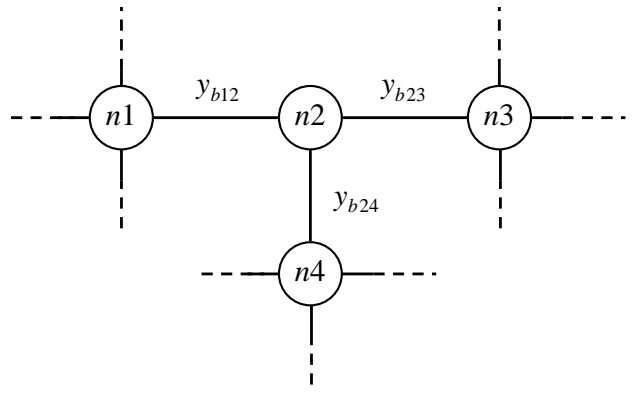

Figura 3: Novo caminho definido por um nó de passagem e três circuitos adjacentes

utilidade (quando apenas um dos ramos $b 12, b 23$ ou $b 24$ é utilizado). As restrições que definem as formas aceitáveis de operação da rede são as seguintes:

$$
\begin{aligned}
& y_{b 12} \leq y_{b 23}+y_{b 24} \\
& y_{b 23} \leq y_{b 12}+y_{b 24} \\
& y_{b 24} \leq y_{b 12}+y_{b 23}
\end{aligned}
$$

A introdução da restrição de igualdade (26) elimina 2 das 4 alternativas de utilização do caminho mostrado na figura 2 que inclui o nó $n 2$, sendo equivalente a eliminar uma variável binária ( $y_{b 12}$ ou $\left.y_{b 23}\right)$. A adição simultânea das restrições (27) elimina 3 das 8 alternativas de utilização do caminho mostrado na figura 3 . No caso geral, um caminho com $n$ nós adjacentes tem $2^{n}$ alternativas de utilização, sendo possível incluir $n$ restrições de desigualdade de novos caminhos para eliminar $n$ destas alternativas de utilização. Embora a redução da complexidade do problema de utilização dos ramos que constituem um novo caminho seja igual a $n / 2^{n}$, observase que nos casos reais $n$ é menor ou igual a 4 ramos. Desta forma, a utilização das restrições de novos caminhos é significativa e contribui para redução da dimensão do problema.

Para os ramos que possuem mais de uma alternativa, no lugar das variáveis $y_{b 12}, y_{b 23}$ e $y_{b 24}$ deve-se utilizar o somatório das variáveis de utilização para cada estágio $t$, ou seja, $\sum_{I \in \Omega_{i}^{F}} y_{i, t}^{F I}$ para os ramos da rede fixa, $\sum_{J \in \Omega_{j}^{R}} y_{j, t}^{R J}$ para os ramos da rede de substituição e $\sum_{K \in \Omega_{k}^{A}} y_{k, t}^{A K}$ para os ramos da rede de adição. Este conceito pode ainda ser estendido para os caminhos que contenham maior número de nós de passagens (dois ou mais), quando aplicado para cada um dos nós de passagem existentes no caminho.

Considerando as três alternativas de investimento para os ramos do novo caminho da figura 2 que são mostradas na tabela 1 , existem $2^{6}=64$ combinações para as seis variáveis binárias de investimento. A imposição das restrições (16) e (17) reduz o número de combinações para $4^{2}$, pois em cada ramo apenas um investimento pode ser selecionado.

Considerando as alternativas de investimento da tabela 1 , en-
Tabela 1: Alternativas de investimento para o caminho da figura 2

\begin{tabular}{|c|c|c|c|}
\hline Ramo & Variável de investimento & $f_{\max }$ & $C$ \\
\hline \multirow{3}{*}{$b 12$} & $x_{b 12}^{1}$ & 2 & 20 \\
\cline { 2 - 4 } & $x_{b 12}^{2}$ & 3 & 30 \\
\cline { 2 - 4 } & $x_{b 12}^{3}$ & 5 & 50 \\
\hline \multirow{3}{*}{$b 23$} & $x_{b 23}^{1}$ & 1,5 & 10 \\
\cline { 2 - 4 } & $x_{b 23}^{2}$ & 2,5 & 20 \\
\cline { 2 - 4 } & $x_{b 23}^{3}$ & 4 & 30 \\
\hline
\end{tabular}

Tabela 2: Combinações de investimentos para o caminho da figura 2

\begin{tabular}{|c|c|c|c|c|c|c|c|c|}
\hline$f_{\max }$ & $x_{b 12}^{1}$ & $x_{b 12}^{2}$ & $x_{b 12}^{3}$ & $x_{b 23}^{1}$ & $x_{b 23}^{2}$ & $x_{b 23}^{3}$ & Custo & Atrativa \\
\hline $\mathbf{0}$ & $\mathbf{0}$ & $\mathbf{0}$ & $\mathbf{0}$ & $\mathbf{0}$ & $\mathbf{0}$ & $\mathbf{0}$ & $\mathbf{0}$ & Sim \\
\hline 0 & 0 & 0 & 0 & 1 & 0 & 0 & 10 & Não \\
\hline 0 & 0 & 0 & 0 & 0 & 1 & 0 & 20 & Não \\
\hline 0 & 0 & 0 & 0 & 0 & 0 & 1 & 30 & Não \\
\hline 0 & 1 & 0 & 0 & 0 & 0 & 0 & 20 & Não \\
\hline $\mathbf{1 , 5}$ & $\mathbf{1}$ & $\mathbf{0}$ & $\mathbf{0}$ & $\mathbf{1}$ & $\mathbf{0}$ & $\mathbf{0}$ & $\mathbf{3 0}$ & Sim \\
\hline $\mathbf{2}$ & $\mathbf{1}$ & $\mathbf{0}$ & $\mathbf{0}$ & $\mathbf{0}$ & $\mathbf{1}$ & $\mathbf{0}$ & $\mathbf{4 0}$ & Sim \\
\hline 2 & 1 & 0 & 0 & 0 & 0 & 1 & 50 & Não \\
\hline 0 & 0 & 1 & 0 & 0 & 0 & 0 & 30 & Não \\
\hline 1,5 & 0 & 1 & 0 & 1 & 0 & 0 & 40 & Não \\
\hline $\mathbf{2 , 5}$ & $\mathbf{0}$ & $\mathbf{1}$ & $\mathbf{0}$ & $\mathbf{0}$ & $\mathbf{1}$ & $\mathbf{0}$ & $\mathbf{5 0}$ & Sim \\
\hline $\mathbf{3}$ & $\mathbf{0}$ & $\mathbf{1}$ & $\mathbf{0}$ & $\mathbf{0}$ & $\mathbf{0}$ & $\mathbf{1}$ & $\mathbf{6 0}$ & Sim \\
\hline 0 & 0 & 0 & 1 & 0 & 0 & 0 & 50 & Não \\
\hline 1,5 & 0 & 0 & 1 & 1 & 0 & 0 & 60 & Não \\
\hline 2,5 & 0 & 0 & 1 & 0 & 1 & 0 & 70 & Não \\
\hline $\mathbf{4}$ & $\mathbf{0}$ & $\mathbf{0}$ & $\mathbf{1}$ & $\mathbf{0}$ & $\mathbf{0}$ & $\mathbf{1}$ & $\mathbf{8 0}$ & Sim \\
\hline
\end{tabular}

tre as 16 combinações possíveis, existem 6 atrativas que são mostradas em destaque na tabela 2 , em função da capacidade $\left(f_{\max }\right)$ e do custo do caminho.

As combinações não-atrativas podem ser eliminadas por intermédio das seguintes restrições:

$$
\begin{gathered}
x_{b 23}^{1} \leq x_{b 12}^{1} \\
x_{b 23}^{2} \leq x_{b 12}^{1}+x_{b 12}^{2} \\
x_{b 23}^{3} \leq x_{b 12}^{2}+x_{b 12}^{3} \\
x_{b 12}^{1}+x_{b 12}^{2}+x_{b 12}^{3}=x_{b 23}^{1}+x_{b 23}^{2}+x_{b 23}^{3}
\end{gathered}
$$




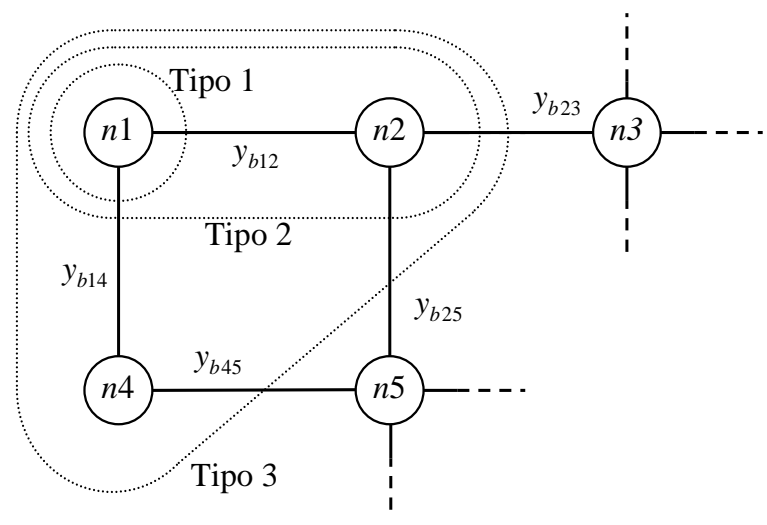

Figura 4: Restrições de cerca em torno do nó $n 1$

\subsubsection{Restrições de cerca}

As restrições de cerca são uma generalização da Lei de Kirchhoff das Correntes e fazem parte de uma metodologia heurística de planejamento da expansão da transmissão denominada "Método de Cerca" (Baldwin et alii, 1960). A figura 4 ilustra os três tipos de cerca que são utilizadas neste artigo: (a) em torno de um nó (Tipo 1); (b) em torno dos nós extremos de um ramo (Tipo 2); (c) em torno de todos os vizinhos de um nó (Tipo 3).

Caso o nó $n 1$ da figura 4 tenha injeção (carga ou geração) diferente de zero, significa que $n 1$ deve ser conectado à rede, sendo a restrição de cerca do Tipo 1 dada por:

$$
y_{b 12}+y_{b 14} \geq 1
$$

Caso exista injeção diferente de zero em algum dos nós extremos do ramo $b 12$ (nós $n 1$ ou $n 2$ ), significa que pelo menos um dos extremos do ramo $b 12$ deve ser conectado à rede, sendo a restrição de cerca do Tipo 2 dada por:

$$
y_{b 14}+y_{b 23}+y_{b 25} \geq 1
$$

Caso exista injeção diferente de zero no nó $n 1$ ou em algum dos seus vizinhos (nós $n 2$ ou $n 4$ ), significa que pelo menos um dos nós $n 1, n 2$ ou $n 3$ deve ser ligado à rede, sendo a restrição de cerca do Tipo 3 dada por:

$$
y_{b 23}+y_{b 25}+y_{b 45} \geq 1
$$

Como observado no caso das restrições de novos caminhos, para os ramos que possuem mais de uma alternativa, no lugar das variáveis $y_{b 12}, y_{b 14}, y_{b 23} \quad y_{b 25} \mathrm{e} y_{b 45}$ deve-se utilizar o somatório das variáveis de utilização para cada estágio $t$.

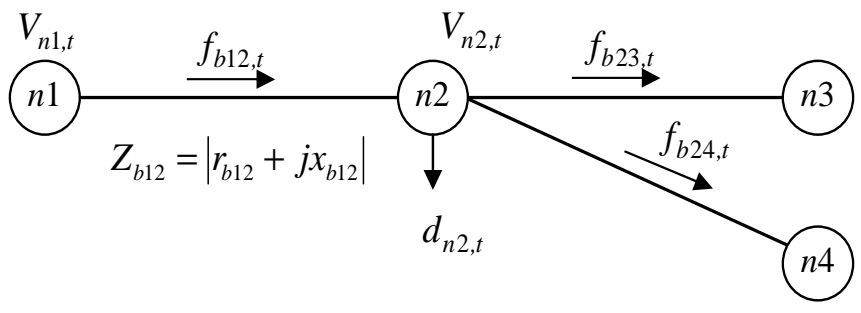

Figura 5: Trecho de rede entre dois nós

\section{REPRESENTAÇÃO DA CARGA E DA REDE}

O modelo de carga e de rede utilizado neste trabalho corresponde a uma adaptação do modelo linearizado de rede apresentado em Romero et alii (2002). No modelo linearizado tradicional, são utilizadas injeções constantes de potência, ângulos de fase das tensões nodais e reatâncias dos ramos; na formulação adotada, ao invés destas grandezas são utilizadas injeções constantes de corrente, magnitudes das tensões nodais e o módulo da impedância dos ramos. Desta forma, a queda de tensão em um determinado trecho de alimentador é dada pelo produto do módulo da impedância $\left(Z_{b 12}\right)$ das linhas pelo fluxo de corrente $\left(f_{b 12}\right)$. Para o trecho ilustrado na figura 5, têm-se as seguintes expressões (em pu) para as Leis de Kirchhoff das Correntes e Tensões, respectivamente:

$$
\begin{gathered}
f_{b 12, t}=d_{n 2, t}+f_{b 23, t}+f_{b 24, t} \\
\Delta V_{b 12, t}=V_{n 1, t}-V_{n 2, t}=Z_{b 12} f_{b 12, t}
\end{gathered}
$$

A restrição (2) é obtida a partir de (32); as restrições (3) a (6) são obtidas a partir de (33). Nas redes analisadas, os erros médios nos valores das magnitudes das tensões nodais obtidas com este modelo aproximado em comparação com a solução do fluxo de carga foram adequados para os propósitos deste trabalho. Além disto, possibilitam que as magnitudes das tensões nodais se relacionem com os fluxos de corrente por intermédio de uma relação linear. Desta forma, a complexidade do modelo de otimização do problema não é aumentada pela inclusão das restrições de queda de tensão.

\section{EXEMPLO}

Para validação da modelagem matemática apresentada, foi utilizada uma rede trifásica fictícia composta por 18 nós (2 subestações e 16 nós de demanda) e 24 ramos, operando em $13800 \mathrm{~V}$. A topologia desta rede é mostrada na figura 6, na qual os retângulos representam as subestações; os círculos os nós onde a demanda é concentrada; os ramos indicados com traço contínuo representam a rede inicial (os ramos indicados com traço simples fazem parte da rede fixa e os ramos indicados com traço duplo são candidatos à substituição) e os ramos indicados com traço pontilhado representam os candi- 
Tabela 3: Dados dos nós da rede de 18 barras

\begin{tabular}{|c|c|c|c|}
\hline \multirow{2}{*}{ Número do nó } & \multicolumn{3}{|c|}{$d[\mathrm{~A}]$} \\
\cline { 2 - 4 } & Estágio 1 & Estágio 2 & Estágio 3 \\
\hline 1 & 50 & 50 & 50 \\
\hline 2 & - & 50 & 50 \\
\hline 3 & - & 50 & 50 \\
\hline 4 & 50 & 50 & 50 \\
\hline 5 & 50 & 50 & 50 \\
\hline 6 & 50 & 50 & 50 \\
\hline 7 & - & 50 & 50 \\
\hline 8 & 50 & 50 & 50 \\
\hline 9 & 50 & 50 & 100 \\
\hline 10 & - & 100 & 150 \\
\hline 11 & 50 & 100 & 150 \\
\hline 12 & 50 & 50 & 50 \\
\hline 13 & 50 & 100 & 150 \\
\hline 14 & - & 50 & 100 \\
\hline 15 & - & 50 & 100 \\
\hline 16 & 50 & 50 & 50 \\
\hline
\end{tabular}

datos à adição (não pertencendo à rede inicial).

A Tabela 3 apresenta a demanda nodal para os três estágios considerados no horizonte de planejamento. As injeções máximas relacionadas com as capacidades existentes das subestações dos nós 17 e 18 são de 500 A para o estágio 1 e 1000 A para os estágios 2 e 3 . Os dados dos ramos encontram-se na Tabela 4, sendo apresentadas nas colunas a capacidade e impedância dos ramos da rede inicial e a capacidade, impedância e custo das alternativas de substituição e adição. $\mathrm{O}$ custo anual de operação e manutenção $\left(O_{i}^{F I}, O_{j}^{R J}\right.$ e $\left.O_{k}^{A K}\right)$ foi considerado igual a 1 para todos os ramos; o custo da energia não suprida $\left(C_{m}^{D}\right)$ foi considerado igual a 100000 para todos os nós de demanda. $\mathrm{O}$ horizonte de planejamento é de quatro anos, dividido em três estágios, sendo os dois primeiros com duração de um ano e o terceiro de dois anos. A taxa de juros adotada foi de $10 \%$ ao ano, sendo os fatores de conversão dos custos de investimento e operação são dados por: $\delta_{1}^{\text {inv }}=\delta_{1}^{\text {oper }}=1, \delta_{2}^{\text {inv }}=\delta_{2}^{\text {oper }}=0,9091$, $\delta_{3}^{\text {inv }}=0,8264$ e $\delta_{3}^{\text {oper }}=1,5778$. Os limites de tensão são $V_{\min }=13110 \mathrm{~V}$ e $V_{\max }=14490 \mathrm{~V}$.

O problema de otimização tem 58 variáveis binárias de investimento (duas opções de cabo para os 5 ramos da rede candidata a substituição; três opções de cabo para os 16 ramos da rede candidata a adição) e 66 variáveis de utilização (uma opção para os 3 ramos da rede pré-existente; o cabo inicial mais duas opções para os 16 ramos da rede candidata a substituição; três opções de cabo para os 16 ramos da rede candidata a adição). Para os três estágios o problema tem 372 variáveis binárias, mas o espaço de busca é drasticamente re-

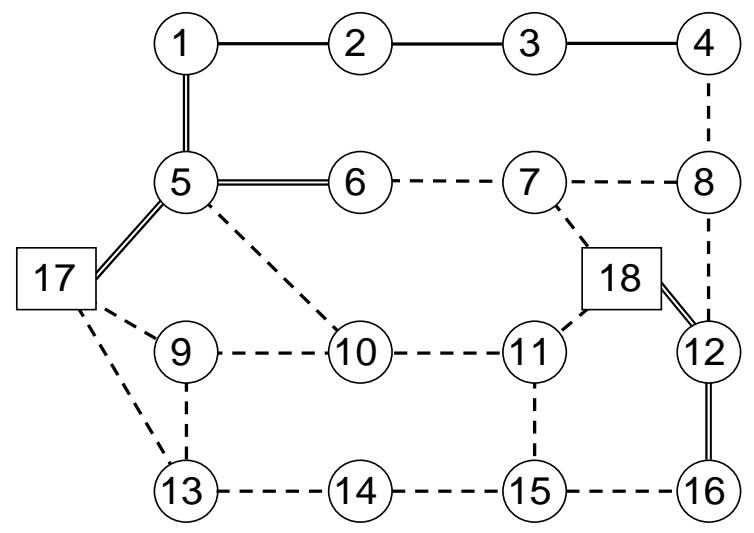

Figura 6: Diagrama da rede de 18 barras

duzido após a introdução das restrições lógicas (16) a (25), passando de $2^{372}$ combinações para aproximadamente $2^{91}$. As restrições adicionais de cerca e de novos caminhos, apresentadas na seção 2.3 , são dependentes da topologia da rede e contribuem ainda mais para a redução do espaço de busca.

São analisadas duas situações: (a) planejamento ano-aano; (b) planejamento multi-estágio. Os resultados apresentados foram obtidos em duas plataformas diferentes: (a) por intermédio do servidor Network-Enabled Optimization System (NEOS, disponibilizado em http://neos.mcs.anl.gov) (Czyzyk et alii, 1998), empregando o solver SCIP (Achterberg, 2004) e o CPLEX 10.10 para solução dos problemas de programação linear (PL), executado em uma estação SunFire V480, com 4 processadores Sparc $31.2 \mathrm{GHz}$, e sistema operacional Solaris 9; (b) utilizando o solver Xpress-MP (Guéret et alii., 2002), versão 2006b, executado em um computador com processador Pentium 4, 2.8 GHz, com 512 MB de RAM, e sistema operacional Windows XP. Ambas implementações estão baseadas no algoritmo enumerativo branch-and-bound, cuja estrutura se baseia em três elementos fundamentais: separação, relaxação e sondagem (Geoffrion e Marsten, 1972). No algoritmo branch-and-bound é realizada a construção de uma árvore de busca, na qual os nós representam os problemas candidatos e os ramos representam as novas restrições que devem ser consideradas. Por intermédio desta árvore, todas as soluções inteiras da região viável do problema são enumeradas de modo implícito ou explícito, o que garante que a solução ótima será encontrada. Na avaliação de cada nó da árvore é resolvido um PL, sendo o esforço computacional empregado na solução do PIM proporcional ao número de nós avaliados.

As soluções ótimas obtidas pelo SCIP e pelo Xpress-MP foram as mesmas e o esforço computacional foi semelhante. Os tempos de processamento apresentados para o SCIP podem apresentar variações significativas em função do carregamento do sistema no momento da execução, pois geral- 
Tabela 4: Dados dos ramos da rede de 18 barras

\begin{tabular}{|c|c|c|c|c|c|c|c|c|c|c|c|c|}
\hline \multicolumn{2}{|c|}{ Nós } & \multicolumn{2}{|c|}{ Inicial } & \multicolumn{3}{|c|}{ Opção 1} & \multicolumn{3}{|c|}{ Opção 2} & \multicolumn{3}{|c|}{ Opção 3} \\
\hline de & para & $f_{\max }[A]$ & $Z[\Omega]$ & $f_{\max }^{1}[A]$ & $Z^{1}[\Omega]$ & $C^{1}[\$]$ & $f_{\max }^{2}[A]$ & $Z^{2}[\Omega]$ & $C^{2}[\$]$ & $f_{\max }^{3}[A]$ & $Z^{3}[\Omega]$ & $C^{3}[\$]$ \\
\hline \multicolumn{13}{|c|}{ Rede pré-existente } \\
\hline 1 & 2 & 250 & 1,0 & - & - & - & - & - & - & - & - & - \\
\hline 2 & 3 & 250 & 1,0 & - & - & - & - & - & - & - & - & - \\
\hline 3 & 4 & 250 & 1,0 & - & - & - & - & - & - & - & - & - \\
\hline \multicolumn{13}{|c|}{ Rede candidata a substituição } \\
\hline 1 & 5 & 250 & 1,0 & 400 & 0,7 & 20 & 500 & 0,5 & 38 & - & - & - \\
\hline 5 & 6 & 250 & 1,0 & 400 & 0,7 & 21 & 500 & 0,5 & 39 & - & - & - \\
\hline 5 & 17 & 250 & 1,0 & 400 & 0,7 & 18 & 500 & 0,5 & 36 & - & - & - \\
\hline 12 & 16 & 250 & 1,0 & 400 & 0,7 & 22 & 500 & 0,5 & 40 & - & - & - \\
\hline 12 & 18 & 250 & 1,0 & 400 & 0,7 & 19 & 500 & 0,5 & 37 & - & - & - \\
\hline \multicolumn{13}{|c|}{ Rede candidata a adição } \\
\hline 4 & 8 & - & - & 250 & 1,0 & 90 & 400 & 0,7 & 110 & 500 & 0,5 & 130 \\
\hline 5 & 10 & - & - & 250 & 1,0 & 92 & 400 & 0,7 & 112 & 500 & 0,5 & 132 \\
\hline 6 & 7 & - & - & 250 & 1,0 & 94 & 400 & 0,7 & 114 & 500 & 0,5 & 134 \\
\hline 7 & 8 & - & - & 250 & 1,0 & 96 & 400 & 0,7 & 116 & 500 & 0,5 & 136 \\
\hline 7 & 18 & - & - & 250 & 1,0 & 300 & 400 & 0,7 & 320 & 500 & 0,5 & 350 \\
\hline 8 & 12 & - & - & 250 & 1,0 & 98 & 400 & 0,7 & 118 & 500 & 0,5 & 138 \\
\hline 9 & 10 & - & - & 250 & 1,0 & 100 & 400 & 0,7 & 120 & 500 & 0,5 & 140 \\
\hline 9 & 13 & - & - & 250 & 1,0 & 102 & 400 & 0,7 & 122 & 500 & 0,5 & 142 \\
\hline 9 & 17 & - & - & 250 & 1,0 & 305 & 400 & 0,7 & 325 & 500 & 0,5 & 355 \\
\hline 10 & 11 & - & - & 250 & 1,0 & 104 & 400 & 0,7 & 124 & 500 & 0,5 & 144 \\
\hline 11 & 15 & - & - & 250 & 1,0 & 106 & 400 & 0,7 & 126 & 500 & 0,5 & 146 \\
\hline 11 & 18 & - & - & 250 & 1,0 & 310 & 400 & 0,7 & 330 & 500 & 0,5 & 360 \\
\hline 13 & 14 & - & - & 250 & 1,0 & 108 & 400 & 0,7 & 128 & 500 & 0,5 & 148 \\
\hline 13 & 17 & - & - & 250 & 1,0 & 315 & 400 & 0,7 & 335 & 500 & 0,5 & 365 \\
\hline 14 & 15 & - & - & 250 & 1,0 & 110 & 400 & 0,7 & 130 & 500 & 0,5 & 150 \\
\hline 15 & 16 & - & - & 250 & 1,0 & 112 & 400 & 0,7 & 132 & 500 & 0,5 & 152 \\
\hline
\end{tabular}

mente são processados diversos problemas simultaneamente.

\subsection{Planejamento ano-a-ano}

Neste caso, realiza-se o planejamento em um estágio de um ano após o outro, utilizando como ponto de partida a solução encontrada na expansão do ano anterior. Na figura 7 apresenta-se os investimentos selecionados (indicados nos respectivos ramos, pelas letras $A 1$, para adição da opção 1 , $R 2$, para substituição pela opção 2), as tensões nodais nos finais dos ramais, as injeções de corrente nas subestações e os custos de cada um dos estágios, correspondendo a um custo total com valor presente de 1488,79. Com o objetivo de reduzir o custo de expansão de cada estágio isoladamente, são realizados alguns investimentos nos estágios 1 e 2 que se tornam obsoletos nos estágios seguintes: adição dos ramos 9-10, 10-11 e 14-15; substituição do ramo 5-17 pela opção
1. A influência dos limites de tensão pode ser verificada pela opção da alternativa de substituição $R 2$ no ramo 12-16. Embora no estágio 3 o fluxo de corrente no ramo 12-16 seja de $300 \mathrm{~A}$, não foi utilizada a opção $R 1$ (mais barata, mas com maior impedância), com capacidade para $400 \mathrm{~A}$, para que a tensão do nó 11 não violasse seu limite inferior.

Para o SCIP, o número de nós avaliados e o tempo de simulação foram de: (a) Estágio 1: 18 nós e 2 segundos; (b) Estágio 2: 543 nós e 3,5 segundos; (c) Estágio 3: 491 nós e 2,4 segundos. Para o Xpress-MP, o número de nós avaliados e o tempo de simulação foram de: (a) Estágio 1: 37 nós e 0,5 segundo; (b) Estágio 2: 1843 nós e 2 segundos; (c) Estágio 3: 1169 nós e 1 segundo. 


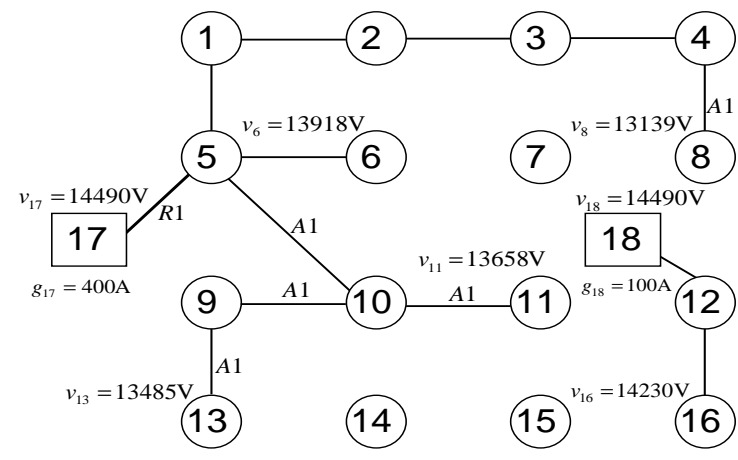

(a) Estágio 1: $c_{1}^{\text {inv }}=506,00$ e $c_{1}^{\text {oper }}=13,00$

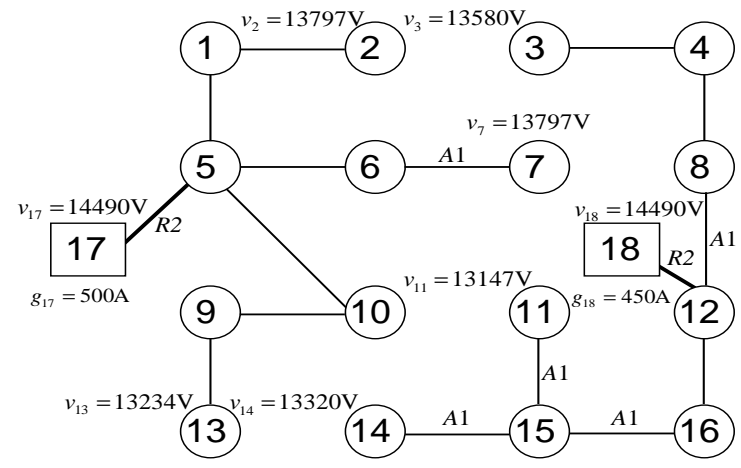

(b) Estágio 2: $c_{2}^{\text {inv }}=593,00$ e $c_{2}^{\text {oper }}=16,00$

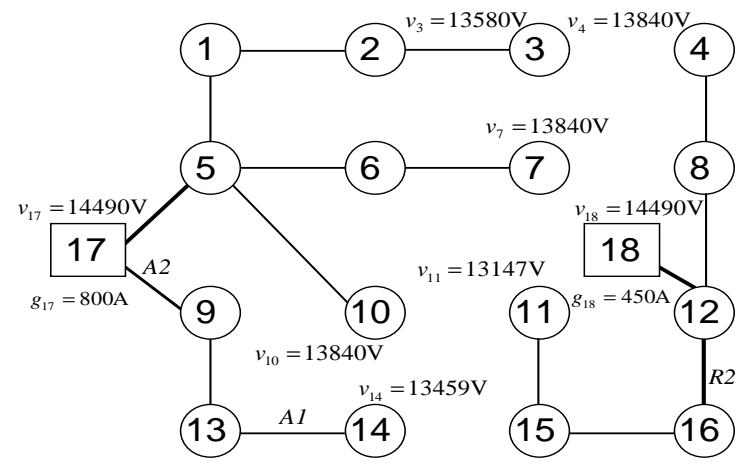

(c) Estágio 3: $c_{3}^{\text {inv }}=473,00$ e $c_{3}^{\text {oper }}=16,00$

Figura 7: Solução expansão ano-a-ano: $C=1488,79$

\subsection{Planejamento multi-estágio}

Neste caso, realiza-se o planejamento considerando os três estágios simultaneamente. A solução obtida e os custos de cada estágio são mostrados na figura 8 , sendo o valor presente igual a 1162,48. Embora o investimento no estágio 1 seja maior que o obtido na expansão ano-a-ano da figura 7 , o custo total é cerca de $23 \%$ menor. A decisão pela instalação de um novo alimentador (ramo 9-17) é antecipada para o primeiro estágio e não são realizados investimentos que se tornam obsoletos, pois o planejamento é realizado com visão

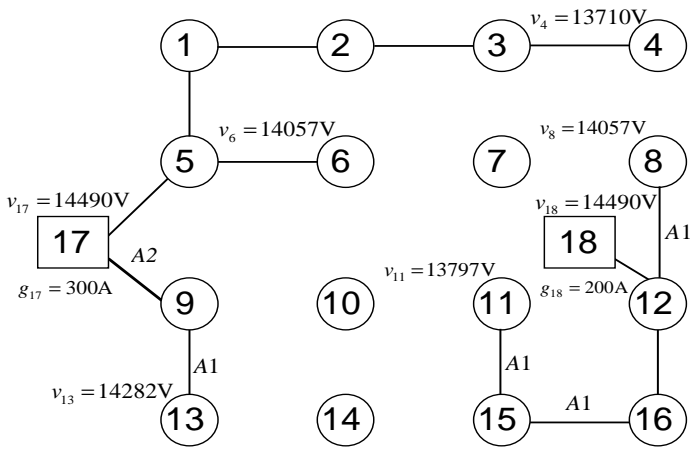

(a) Estágio 1: $c_{1}^{\text {inv }}=743,00$ e $c_{1}^{\text {oper }}=13,00$

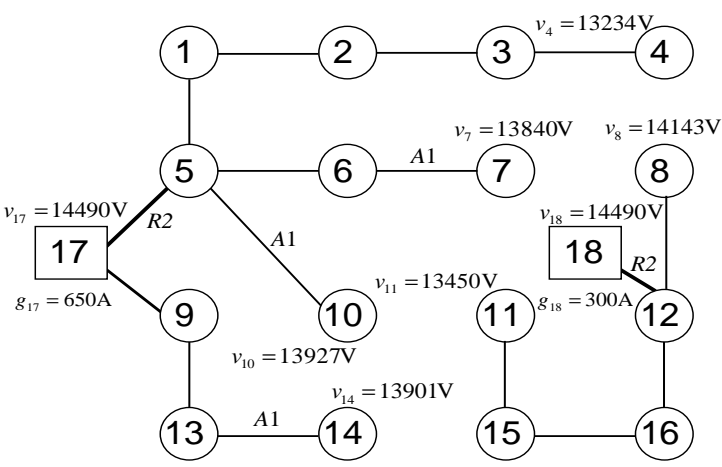

(b) Estágio 2: $c_{2}^{\text {inv }}=367,00$ e $c_{2}^{\text {oper }}=16,00$

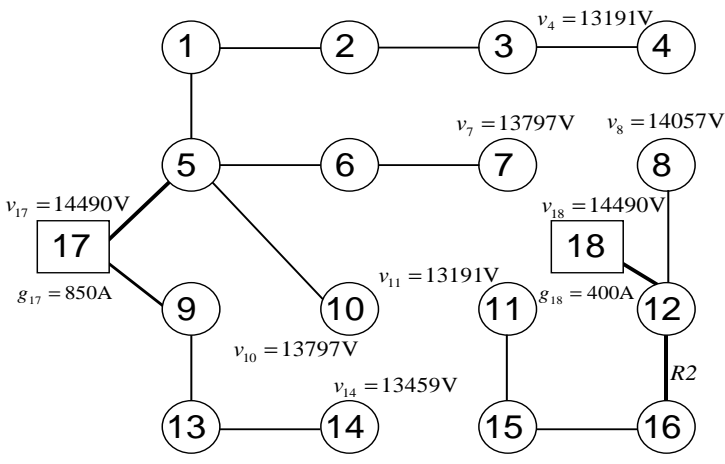

(c) Estágio 3: $c_{3}^{\text {inv }}=40,00$ e $c_{3}^{\text {oper }}=16,00$

Figura 8: Solução expansão multi-estágio: $C=1162,48$

de longo prazo.

Para obtenção da solução com o SCIP foram avaliados 35224 nós em 713 segundos. Para o Xpress-MP foram avaliados 28927 nós em 143 segundos.

\section{CONCLUSÕES}

O modelo multi-estágio de otimização apresentado neste trabalho foi concebido de forma a ser abrangente e flexível. 
Neste modelo foi possível considerar os seguintes tipos de alterações na rede de distribuição: (a) mudança de bitola dos condutores da rede pré-existente (substituição); (b) inserção de novos trechos de rede com opção por diferentes bitolas (adição); (c) não utilização temporária ou permanente (remoção) de trechos de alimentadores (d) instalação de novas subestações; (e) ampliação das subestações existentes. Na função objetivo foram considerados os custos de investimento e de operação, incluindo o custo de operação e manutenção dos alimentadores da rede.

O fato de permitir realizar o planejamento em múltiplos estágios e a possibilidade de representação de diferentes alternativas de alteração permite que se obtenham custos finais inferiores aos obtidos pelos modelos mais simples, pois é possível representar horizontes de planejamento de longo prazo, tirando proveito pela antecipação de investimentos que serão importantes no futuro. Para a rede apresentada no exemplo, houve uma significativa redução de custo (22\%) quando considerado o planejamento em múltiplos estágios.

Foram consideradas as principais restrições do problema real que são a capacidade dos condutores, o limite de queda de tensão e a manutenção da radialidade da rede. Para garantir que a configuração obtida seja sempre radial, pode ser necessário acrescentar restrições com informações específicas sobre a topologia da rede em análise. Considerando a diversidade de situações envolvidas, esta tarefa pode ser relativamente complexa. Neste trabalho foi utilizada uma estratégia mais simples que consiste em limitar o total de novos ramos ativos em cada etapa a um número menor ou igual ao número de nós da rede nesta etapa, conforme expressão (25). Nos testes executados, a restrição (25) em conjunto com as restrições adicionais (novos caminhos e cerca) foi suficiente para que sempre fossem obtidas soluções radiais.

A simplificação empregada na representação da Segunda Lei de Kirchhoff permitiu estabelecer um modelo de otimização com todas as restrições lineares, por intermédio do modelo linear disjuntivo. O modelo linear resultante foi resolvido através de um programa de otimização baseado no método branch-and-bound, permitindo obter a solução ótima do problema de expansão. Embora o problema possa apresentar um grande número de variáveis binárias, a introdução das restrições lógicas - (16) a (25) - e das restrições adicionais - (26) a (31) - reduziu de forma significativa o espaço de busca, tornando o problema inteiro misto computacionalmente tratável.

\section{AGRADECIMENTOS}

O desenvolvimento deste trabalho foi parcialmente apoiado pela Companhia Estadual de Energia Elétrica (CEEE), através de seus programas P\&D ANEEL. Os autores agradecem a Tobias Achterberg pela disponibilização do solver SCIP (Achterberg, 2004), utilizado nas simulações apresentadas neste trabalho.

\section{REFERÊNCIAS}

Achterberg, T. (2004). SCIP - A framework to integrate constraint and mixed integer programming, Zuse Institute Berlin, disponível em julho de 2006 em http://www.zib.de/Publications/abstracts/ZR-04-19/

Aoki, K., Nara, K., Satoh, T., Kitagawa, M. e Yamanaka, K. (1990). New approximate optimization method for distribution system planning, IEEE Trans. on Power Systems, Vol. 5, No.1, pp. 126-132.

Augugliaro, A., Dusonchet, L. e Sanseverino, E.R (2002). An evolutionary parallel tabu search approach for distribution systems reinforcement planning, Advanced Engineering Informatics, No. 16, pp. 205-215.

Bahiense, L., Oliveira, G.C., Pereira, M. e Granville, S. (2001). A mixed integer disjunctive model for transmission network expansion, IEEE Trans. on Power Systems, Vol. 16, No. 3, pp. 560-565.

Baldwin, C.J., DeSalvo, C.A., Hoffman, C.H. e Ku, W.S. (1960). A model for transmission planning by logic, AIEE Trans. Power Apparatus and Systems, Vol. PAS78, pp. $1638-1645$.

Boulaxis, N.G. e Papadopoulos, M.P. (2002). Optimal feeder routing in distribution system planning using dynamic programming technique and GIS facilities, IEEE Trans. on Power Delivery, Vol. 17, No. 1, pp. 242-247.

Czyzyk, J., Mesnier, M.P. e Moré, J.J. (1998). The NEOS server, IEEE Computational Science and Engineering, Vol. 5, No. 3, pp. 68-75.

Díaz-Dorado, E., Cidrás, J. e Míguez, E. (2002). Application of Evolutionary Algorithms for the Planning of Urban Distribution Networks of Medium Voltage, IEEE Trans. on Power Systems, Vol. 17, No. 3, pp. 11511159.

Díaz-Dorado, E. e Pidre, J.C. (2004). Optimal planning of unbalanced networks using dynamic programming optimization, IEEE Trans. on Power Systems, Vol. 19, No. 4, pp. 2077-2085.

El-Khattam, W., Hegazy, Y.G. e Salama, M.M.A. (2005). An integrated distributed generation optimization model for distribution system planning, IEEE Trans. on Power Systems, Vol. 20, No. 2, pp. 1158-1165. 
Farrag, M.A., El-Metwally, M.M. e El-Bages, M.S. (1999). A new model for distribution system planning, Electrical Power \& Energy System, Vol. 21, pp. 523-531.

Geoffrion, A.M. e Marsten, R.E. (1972). Integer programming algorithms: a framework and state-of-the-art survey, Manage Sci., Vol. 18, No. 9, pp. 465-491.

Gómez, J.F., Khodr, H.M., Oliveira, P.M., Ocque, L., Yusta, J.M., Villasana, R. e Urdaneta, A.J. (2004). Ant colony system algorithm for the planning of primary distribution circuits, IEEE Trans. on Power Systems, Vol. 19, No. 2, pp. 996- 1004.

Gönen, T. e Foote, B.L. (1982). Mathematical dynamic optimization model for electrical distribution system planning, Electrical Power \& Energy Systems, Vol. 4, No. 2, pp. 129-136.

Guéret, C., Prins, C. e Sevaux, M. (2002). Applications of Optimization with Xpress-MP, Dash Optimization Ltd., United Kingdom, p. 349.

Khator, S.K. e Leung, L.C. (1997). Power distribution planning: a review of models and issues, IEEE Trans. on Power Systems, Vol. 12, No. 3, pp. 1151-1159.

Lakervi E. e Holmes, E.J. (1995). Electricity distribution network design, 2nded., IEE Power Series 21, London, Peter Peregrinus Ltd., p. 325.

Míguez, E., Cidrás, J., Díaz-Dorado, E. e García-Dornelas, J.L. (2002). An improved branch-exchange algorithm for large-scale distribution network planning, IEEE Trans. on Power Systems, Vol. 17, No. 4, pp. 931-936.

Miranda, V., Ranito, J.V. e Proença, L.M. (1994). Genetic algorithms in optimal multistage distribution network planning, IEEE Trans. on Power Systems, Vol. 9, No. 4, pp. 1927-1933.

Monticelli, A., Santos, A., Pereira, M.V.F., Cunha, S., Praça, J.G. e Park, B. (1982). Interactive transmission network planning using least-effort criterion, IEEE Trans. Power Apparatus and Systems, Vol. PAS-101, No. 10, pp. 3919-3925.

Nara, K., Satoh, T., Kuwabara, H., Aoki, K., Kitagawa, M. e Ishihara, T. (1992). Distribution systems expansion planning by multi-stage branch exchange, IEEE Trans. on Power Systems, Vol. 7, No. 1, pp. 208-214.

Nara, K., Hayashi, Y., Muto, S. e Tuchida, K. (1998). A new algorithm for distribution feeder expansion planning in urban area, Electric Power System Research, Vol. 46, pp.185-193.
Paiva, P.C., Khodr, H.M., Domínguez-Navarro, J.A., Yusta, J.M. e Urdaneta, A.J. (2005). Integral planning of primary-secondary distribution systems using mixed integer linear programming, IEEE Trans. on Power Systems, Vol. 20, No. 2, pp. 1134-1143.

Parada, V., Ferland, J.A., Arias, M. e Daniels, K. (2004). Optimization of electrical distribution feeders using simulated annealing, IEEE Trans. on Power Delivery, Vol. 19, No. 3, pp. 1135-1141.

Ranjan, R., Venkatesh, B. e Das, D. (2002). A new algorithm for power distribution system planning, Eletric Power System Research, Vol. 62, No. 1, pp. 55-65.

Ramírez-Rosado, I. J. e Gönen, T. (1991). Pseudodynamic planning for expansion of power distribution systems, IEEE Trans. Power Systems, Vol. 6, No. 1, pp. 245-254.

Ramírez-Rosado, I.J. e Bernal-Augustín, J.L. (2001). Reliability and costs optimization for distribution networks expansion using an evolutionary algorithm, IEEE Trans. on Power Systems, Vol. 16, No. 1, pp. 111-118.

Romero, R., Monticelli, A., Garcia, A. e Haffner, S. (2002). Test systems and mathematical models for transmission network expansion planning, IEE Proc.-Gener. Transm Distrib., Vol. 1491, No. 1, pp. 27-36.

Ponnavaikko, M, Prakasa Rao, K.S. e Venkata, S.S. (1987). Distribution system planning through a quadratic mixed integer programming approach, IEEE Trans. on Power Delivery, Vol. 2, No. 4, pp. 1157-1163.

Sun, D.I., Farris, D.R., Cote, P.J., Shoults, R.R. e Chen, M.S. (1982). Optimal distribution substation and primary feeder planning via the fixed charge network formulation, IEEE Trans. Power Apparatus and Systems, Vol. PAS101, No. 3, pp. 602-609.

Temraz, H.K. e Quintana, V.H. (1993). Distribution system expansion planning models: an overview, Electric Power Systems Research, Vol. 26, pp. 61-70.

Vaziri, M., Tomsovic, K. e Bose, A. (2004a). A direct graph formulation of the multistage distribution expansion problem, IEEE Trans. on Power Delivery, Vol.19, No. 3, pp. 1335-1341.

Vaziri, M., Tomsovic, K. e Bose, A. (2004b). Numerical analyses of a direct graph formulation of the multistage distribution expansion problem, IEEE Trans. on Power Delivery, Vol.19, No. 3, pp. 1348-1354.

Willis, H.L. (2004). Power Distribution Planning Reference Book - Second Edition Revised and Expanded, Marcel Dekker, New York, p. 1217. 